\title{
High Fluoride Intake and Low Calcium Alongwith Genetic Predisposition May Lead to Bone Weakness and Deformation
}

\author{
Mahendra Kumar Verma ${ }^{1}$, Ravinder Pal Singh ${ }^{2}$, Manoj G Tyagi ${ }^{* 1}$ \\ ${ }^{1}$ Department of Paramedical Technology, NIMS University, Jaipur, Rajasthan \\ ${ }^{2}$ College of Pharmacy, NIMS University, Jaipur, Rajasthan
}

Author for Correspondence: Dr Manoj G Tyagi; tyagi257@yahoo.in

Received 09 April 2019;

Accepted 20 April 2019;

Published 29 April 2019

\begin{abstract}
Fluoride affects bone strength, by replacing hydroxyl ions in bone crystals to form fluoroapatite, and increasing both the osteoblast and osteoclast activity. Daily intake of naturally fluoridated water, is inevitable in many areas and countries throughout the world, it remains a major health issue as it has been suggested that fluorides may paradoxically aggravate osteoporosis and increase osteoporotic fractures and several epidemiological studies have been published showing that there is an increased rate of bone fractures i.e mostly hip fractures in older people in living in fluoridated areas. This study was carried out to ascertain the fact that high fluoride intake and low calcium may have on bone deformities in patients.
\end{abstract}

\section{Introduction}

The fluoridation debate highlights the dynamics of science and power. Till date, the dominant narrative has been that water fluoridation is safe and effective, with advocates claiming strong scientific support and the endorsement of the practice by major dental and public health bodies as evidence of its effectiveness ${ }^{[1]}$. This is despite key questions about the efficacy and effectiveness of ingested fluoride, concerns about safety, and questions about ethics and legality producing a debate that is a potent mixture of scientific, professional, corporate, and ethical arguments ${ }^{[2-5]}$. Recentresearch papersprovides a reasoned assessment on the magnitude of the main positive impact of fluoride ingestion on human health i.e., prevention of dental cariescompared with the established and potential adverse impacts (Refer Fig.1). In particular, it raises questions about what an acceptable safety margin should be for ingested fluoride and questions why normal rules of safety normally applied to assessments of harm and benefit are not applied to water fluoridation.Almost60 years have elapsed since fluoride was proposed for treating the bone mineral disorder osteoporosis. Although several prospective studies have shown that fluoride salts are effective in increasing cancellous bone mass in osteoporotic patients, increasing concern has arisen over the quality of the newly synthesised bone. In fact, clinical studies have suggested that fluoride therapy increases spinal bone mineral density but does not decrease the rate of vertebral fractures. Moreover, some studies have even suggested a trend to an increase in the incidence of fractures in the appendicular skeleton ${ }^{[6,7]}$. Healthy bones in the adult and elderly population are dependent on the development during the younger years of a healthy bone structure and an adequate peak bone mass.

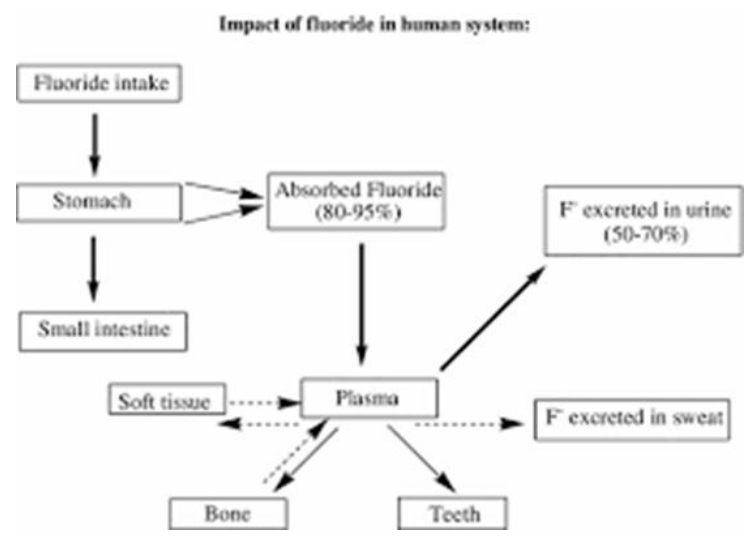

Fig.1 Fluoride distribution in human body
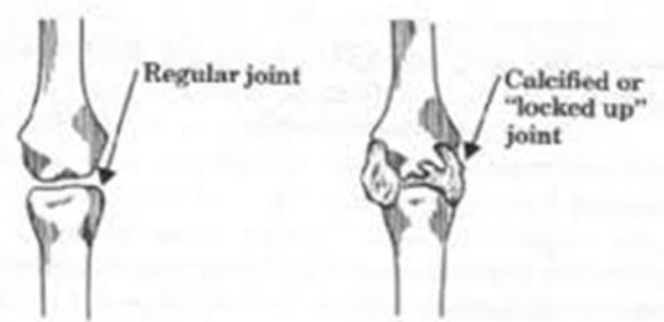

Normal

Fluoride-Treated 
Skeletal fluorosis and factors affecting it: Skeletal fluorosis is a chronic metabolic bone and joint disease caused by ingesting large amounts of fluoride ${ }^{[8]}$. Excessive fluoride intake can disrupt bone turnover, influences the differentiation of osteoblasts and osteoclasts and resulting in the development of bone lesions ${ }^{[9,10]}$ (Refer Fig.2). This eventually causes an imbalance of bone formation and bone resorption. Skeletal fluorosis may be affected by multiple factors for eight risk for developing skeletal fluorosis, and the course the disease will take, can be strongly influenced by the presence or absence of predisposing factors, which include impaired kidney function; dietary deficiencies; genetic predisposition; gastric acidity; physical/repetitive stress; age; and pregnancy/lactation. Individuals with kidney disease have decreased ability to excrete fluoride in urine and are at risk of developing fluorosis even at normal recommended limit of 0.7 to $1.2 \mathrm{mg} / \mathrm{l}^{[11,12]}$. Previous studies in mice and humans, as well as epidemiological studies, have demonstrated that severity of dental fluorosis cannot be explained simply by the amount of fluoride in the tooth structure, indicating that genetics (susceptiblity to fluoride) plays an important role in dental fluorosis severity. Based on that, one can infer that in individuals ingesting the same amount of fluoride, the dental fluorosis severity will be related to and/or based on individual susceptibility to fluoride. This study helped 1) to establish the existence of genetic predisposition to fluorosis and develop criteria for estimating it, and 2) to prove that predisposition to fluorosis was associated with the same dermatoglyphic features in the workers of both industrial groups suggest that predisposition to fluorosis (chronic toxicity) is biochemically mediated and genetically determined ${ }^{[13,14]}$. A study by Simon et al depicted that high fluoride and low calcium intake attributed to alveolar bone $\operatorname{loss}^{[15]}$. This would explain the marked variation in fluorosis prevalence in areas with comparable levels of fluoride intake and the selectivity of the disease within the same area. Further studies are necessary to elucidate the complex interaction between calcium, iodine, sex hormones, vitamins and fluoride ions. Skeletal bone undergoes modeling to help it in adapting to changes in biomechanical forces and remodeling to remove old, micro-damaged bone and replace it with new bone which is mechanically stronger that helps in maintaining bone strength $^{[16]}$. Long bones like the radius, femur and tibia are composed of a hollow shaft or diaphysis, flared, cone-shaped metaphyses below the growth plates and rounded epiphyses above the growth plates. The diaphysis is formed from dense cortical bone, while the metaphysis and epiphysis are formed from trabecular meshwork bone which is surrounded by a thin layer of dense cortical bone ${ }^{[16]}$. In a study that was done during the period of 1978 to 1982, and included 4,121 men and 16,272 women who were aged 45 years and older suffered from hip fracture, living in 39 districts in England and Wales, and were also part of the British Regional Heart Study. When analyzing the data, hip fracture discharge rates from the county district were correlated with water fluoride concentration. However, hip fracture discharge rates and water fluoride levels were not associated $(r=0.16, p=0.34)$. Reanalysis of the data was conducted by applying a population regression model, where significant positive correlation was found between fluoride concentration and discharge rates for hip fractures $(\mathrm{r}=0.41, \mathrm{p}=0.009)$. Vitamin $\mathrm{D}$ plays an indirect role in this process. The presence of vitamin D isessential for maintaining adequate concentrations of calcium and phosphate for bone mineralization. This is achieved by stimulating the absorption of calcium and phosphate from the gastrointestinal tract. The mineral component of bone is responsible for the rigidity and strength of the bone. The organic matrix gives it its elasticity and flexibility.
The extracellular bone matrix protein is composed of 85 to $90 \%$ collagenous proteins, mainly type I collagen ${ }^{[17,18]}$. A study conducted in children found that Vitamin D3 reversed the clinical features of fluorosis amongst children.

Mechanism of Fluoride action on the bones: Fluoride concentration in bone is not consistent throughout the body. In the long bones, the highest concentrations are found at the periosteal region. A sharp decline in fluoride concentration is noted within a few millimeters of the periosteal surface and the concentration increases slightly at the end steal region ${ }^{[19]}$. Fluoride concentrations also vary in different bone components, with higher fluoride levels found in the trabecular bone compared to the cortical bone ${ }^{[19]}$.

Analytical Methods: Fluoride is usually determined by means of an ion-selective electrode, which makes it possible to measure the total amount of free and complex-bound fluoride dissolved in water. The method can be used for water containing at least 20 $\mu \mathrm{g} / \mathrm{litre}$. For rainwater in which fluoride was present at a concentration of $10 \mu \mathrm{g} /$ litre, a detection limit of $1 \mu \mathrm{g} /$ litrehas been reported $^{[20]}$. A method using a fluoride-selective electrode and an ion analyser to determine fluoride at levels of $0.05-0.4 \mathrm{mg} / \mathrm{litre}$ has been described. Fluoride can be detected in water, milk and dairy products using these techniques ${ }^{[21]}$.

Genetic studies and predisposition: Genetic predisposition could be a factor in the progression of many diseases and also fluoride induced skeletal deformations however other key factors associated in this regard like the parasitic infections again due to genetic factors may lead to skeletal fluorosis ${ }^{[22]}$. Genetic studies of some mutant nematodes have led to the identification of novel fluorideresistant (flr) genes, flr1, flr3, and flr4. The flr1 gene encodes an ion channel belonging to the degenerin/epithelial sodium channel superfamily, which regulates defecation rhythm. The flr4 gene encodes a predicted Ser/Thr protein kinase and, like flr-1, appears to control rhythmic activities in Caenorhabditiselegans ${ }^{[23,24]}$. The flr-4 gene is closely related to the human SOK1 gene, a Ste20 protein kinase of the germinal center kinase (GCK) family. The flr3 gene remains to be characterized.

\section{Conclusions}

This review argues about the modest benefits of chronically ingested fluoride in caries but it adversely affecting the bone morphology and integrity. The high fluoride content in drinking water are thoroughly counterbalanced by its established and potential diverse adverse impacts on human health. Due to the abundance of this chemical, it is little surprising that humans ingest or inhale fluoride from a variety of sources. In the Hippocratic treatise titled Epidemics, the ethical principle in relation to controlling disease Primum non nocere ("do good or to do no harm") was emphasised. This principle is, at best, not being fully observed in relation to fluoride-centred prevention interventions, given the established and potential harms currently attributed to fluoride in humans. It appears that chronic high fluoride levels, low calcium in water and diet along with genetic predisposition lead to skeletal fluorosis and further studies are needed to completely establish this interactive relationship leading to skeletal deformation.

\section{References}

[1] P. E. Petersen and M. A. Lennon, "Effective use of fluorides for the prevention of dental caries in the $21 \mathrm{st}$ 
century: the WHO approach," Community Dentistry and Oral Epidemiology, vol. 32, no. 5, pp. 319-321, 2004.D. Shaw, "Weeping and wailing and gnashing of teeth: the legal fiction of water fluoridation," Medical Law International, vol. 12, no. 1, pp. 11-27, 2012.

[2] N. Awofeso, "Ethics of artificial water fluoridation in Australia," Public Health Ethics, vol. 5, no. 2, pp. 161172, 2012.

[3] S. Peckham, "Slaying sacred cows: is it time to pull the plug on water fluoridation?" Critical Public Health, vol. 22, no. 2, pp. 159-177, 2012.

[4] P. Connett, B. James, and M. Spedding, The Case against Fluoride: How Hazardous Waste Ended up in Our Drinking Water and the Bad Science and Powerful PolItics That Keep It There, Chelsea Green Publishing, 2010.

[5] Schnitzler CM, Wing JR, Gear KA, Robson HJ. Bone fragility of the peripheral skeleton during fluoride therapy for osteoporosis. ClinOrthop;261:268-75, 1990.

[6] Hedlund LR, Gallagher JC. Increased incidence of hip fracture in osteoporotic women treated with sodium fluoride. J Bone Miner Res;4:223-5,1989.

[7] Lee JR. Fluoridation and hip fracture: according to the National Research Council Report "Health Effects of Ingested Fluoride”. Fluoride;26:274-7, 1993.

[8] Anand JK, Roberts JT. Chronic fluorine poisoning in man: a review of literature in English (1946-1989) and indications for research. Biomedicine \& Pharmacotherapy 44: 417-420,1990.

[9] Chen Y, Yan W, Hui X. Treatment and prevention of skeletal fluorosis. Biomed Environ. Sci; 30: 147-149, 2017.

[10] Gupta N, Gupta N, Chhabra P. Imaging diagnosis: Dental and skeletal fluorosis. Prem J. 20: 107-116. 2016.

[11] Bansal R, Tiwari SC.Back pain in chronic renal failure. Nephrology Dialysis Transplantation 21:23312332,2006.

[12] Polzik EV, et al. A method for estimating individual predisposition to occupational fluorosis. Fluoride 27: 194-200,1994.
[13] Lavryashina MB, et al. A study of the genetic basis of susceptibility to occupational fluorosis in aluminum industry workers of Siberia. Russian Journal of Genetics 39: 823-827,2003.

[14] Saha PK, Saha D. The genetic influence in fluorosis. Environ. Toxicol.Pharmacol.56, 157-162, 2017.

[15] Simon M, Beil F, Riedel C. Deterioration of teeth and alveolar bone loss due to chronicenvironmental highlevel fluoride and low calcium exposure. Clin Oral Investig; 20 (9): 2361-2370, 2016.

[16] Reem Oweis.Associations between fluoride intakes, bone outcomes and dental fluorosis. $\mathrm{PhD}$ thesis submitted to the University of Iowa, 2018.

[17] S.M. Levy, J.J. Warren, K. Phipps, E. Letuchy, B. Broffitt, J. Eichenberger-Gilmore, T.L. Burns, G. Kavand, K.F. Janz, J.C. Torner and C.A. Pauley. Effects of Life-long Fluoride Intake on bone Measures of Adolescents: A Prospective cohort study.J Dent Res 93(4), 353-359, 2014.

[18] Gupta SK, Gupta RC and Seth AK. Reversal of clinical and dental fluorosis. Indian Pediatrics, 31, 439-443, 1994.

[19] Whitford G. Intake and metabolism of fluoride. Adv Dent Res; 8: 5-154, 1994.

[20] Ahmed R, Hussain M, TanwirR, Queirishi SA. Monitoring of fluoride and iodide levels in drinking water using ion selective electrode. The Nucleus; 41: 51 $58,2004$.

[21] E Ocak and S Kose. Determination of fluoride in water, milk and dairy products. Fluoride, 51(2), 182-192, 2018.

[22] Iwasaki K, Thomas JH. Genetics in rhythm. Trends Genet 13:111-115, 1997.

[23] Sura I, Kondo K, Amano T, Ishihara T, Kawakami M. Isolation, characterization and epistasis of fluorideresistant mutants of Caenorhabditiselegans. Genetics 136:145-154, 1994.

[24] Take-Uchi M, Kawakami M, Ishihara T, Amano T, Kondo K, Katsura I. An ion channel of the degenerin/epithelial sodium channel superfamily controls the defecation rhythm in Caenorhabditiselegans. Proc Natl Acad Sci USA 95:11775-11780, 1998. 